\title{
APRENDIZAJE SIGNIFICATIVO DE VALORACIÓN AL GERONTE DESDE OREM, PROMOVIDO POR UN AMBIENTE B-LEARNING
}

\author{
SIGNIFCANT LEARNING FROM ASSESSMENT TO OREM ELDERLY PROMOTED BY A \\ B-LEARNING ENVIRONMENT
}

\author{
Adriana Hernández Bustos* y Ruby Marcela Torres Rivera**
}

\begin{abstract}
RESUMEN
Con el interés de promover aprendizaje significativo en los estudiante de enfermería se desarrolla la presente investigación cualitativa a la que se incorporan las tecnologías de la información y comunicación, en el aprendizaje de la valoración de los requisitos de autocuidado universales: soledad e interacción social y prevención de peligros para la vida, el funcionamiento y bienestar humano del adulto mayor, propuestos por Dorotea Orem. Los principales resultados revelaron que incluir un ambiente B-learning, en el núcleo temático "cuidado de la enfermería en la adultez y senectud", tuvo un gran aporte en el aprendizaje significativo de los dos requisitos de autocuidado por parte de los estudiantes de sexto semestre de enfermería de la Universidad de Cundinamarca, al favorecer la motivación y un uso más flexible del tiempo, lo que les permitió ahondar en conocimientos y promover el pensamiento crítico, de manera que al realizar la valoración práctica de estos requisitos, cumplieron con lo esperado implementando un plan de cuidados contextualizado al geronte.
\end{abstract}

Palabras clave: Aprendizaje significativo, Dorotea Orem, B-learning, geronte, Valoración.

\begin{abstract}
In the interest of promoting meaningful learning in nursing student this qualitative research is conducted; the technologies of information and communication are incorporated in the assessment of learning universal self-care requirements: solitude and social interaction and prevention of hazards to life, functioning and human well-being of the elderly, given by Dorotea Orem. The main results of the research conducted with students of sixth semester of nursing at the University of Cundinamarca, revealed to include a B-learning environment, the "nursing care in adulthood and old age" thematic core, had a great contribution in meaningful learning of the two self-care requirements, to encourage motivation and a more flexible use of time, allowing students to deepen knowledge and promote critical thinking, so that when you make the practical assessment of these requirements, met with expectations implementing a plan of care to geronte contextualized.
\end{abstract}

Keywords:Meaningful learning, Orem, B-Learning Environment, Elderly, Assessment.

Fecha de recepción: Marzo 4 de 2014 / Fecha de aceptación: Julio 10 de 2014

Tipología: Artículo de Investigación Científica y Tecnológica

Para citar este artículo: Hernández, B. A., \& Torres, R. R. (2014). Aprendizaje significativo de valoración al geronte desde orem, promovido por un ambiente b-learning. Praxis, Vol. 10, 37 - 47

\footnotetext{
*Magister en Tecnologías de la Información Aplicadas a la Educación 2014 Universidad de Cundinamarca, Colombia. E-mail: adrianaher7714@hotmail.com. **Magister en Tecnologías de la Información Aplicadas a la Educación 2014 IED los Alpes, Colombia. E-mail: marcelalaguerrera@gmail.com.
} 


\section{INTRODUCCION}

Este trabajo se planteó ante la necesidad evidenciada por los profesionales colombianos en enfermería, de brindar cuidados a una población que está envejeciendo como fenómeno demográfico predominante de estos tiempos (Stanley, Blair \& Gauntlett, 2009). Situación que exige gran apropiación conceptual en los estudiantes, para promover la salud a partir de acciones preventivas que favorezcan el cumplimiento de los requisitos de Autocuidado propuestos por Dorotea Orem.

Dominio que no presentaron a satisfacción los estudiantes de sexto semestre enfermería de la Universidad de Cundinamarca (Colombia). Deducción a la que se llegó luego de la revisión de 30 registros seleccionados de forma aleatoria, diligenciados entre los años 2007 y 2012 por estudiantes de dicha facultad; en la que solo el 16,7 $\%$ cumplió con el protocolo de diligenciamiento $y$, por ende, en el planteamiento del proceso de atención de enfermería dentro de la integralidad requerida para satisfacer las necesidades de los adultos mayores.

Aprender e implementar la Teoría de déficit de autocuidado de Dorotea Orem en el proceso de atención integral, es importante porque permite una relación de ayuda entre el adulto mayor, su familia y el futuro enfermero, al tener: "En cuenta la relación que existe entre las acciones que un individuo necesita hacer o requiere para mantener su vida, su salud y bienestar y la habilidad de dicho individuo para realizar dichas acciones”. (Scarborough general Hospital, 1984, p.3-4). Lo cual resulta fundamental para el adulto mayor, quien por su ciclo de vida puede estar limitado, bien sea por la incapacidad de satisfacer sus necesidades de autocuidado, o por no poseer la habilidad necesaria para ello; aspectos que justifican la necesidad de la atención del enfermero.

Dentro de los ocho requisitos de autocuidado universales propuestos por Dorotea Orem, solo fueron implementados dos: soledad e interacción social y prevención de peligros para la vida el funcionamiento y bienestar humano; tal selección obedeció a la relevancia de los mismos en el cuidado del geronte y a la falta de información detallada, lo cual no permitía que los estudiantes dimensionaran todos los aspectos que incluían estos dos requisitos.

En la búsqueda de establecer herramientas alternas, que facilitaran el proceso de aprensión del conocimiento de dichos requisitos, se comprendió la necesidad de determinar: ¿Cuál era el aporte que hace una estrategia didáctica centrada en un ambiente b-learning basado en aprendizaje significativo, a la valoración práctica realizada por estudiantes de sexto semestre de enfermería, para los requisitos de soledad e interacción social y prevención de peligros para la vida el funcionamiento y bienestar humano del adulto mayor desde la teoría de Dorotea Orem?. Pregunta problema de la investigación, que se resolvió a partir del desarrollo de los siguientes objetivos específicos:

- Diseñar un ambiente B-learning con 2 OVAS (Objetos virtuales de Aprendizaje), utilizando como estrategia didáctica el uso de animaciones y organizadores previos.

- Identificar la relación entre el uso del ambiente B-learning y las categorías disciplinares de valoración y cuidado.

- Determinar la relación entre el uso del ambiente B-learning y las categorías de motivación y el aprendizaje significativo.

Para dar respuesta a estos, la solución se planteó, desde lo que significa aprender con el apoyo o uso de las tecnologías de la información y la comunicación (también conocidas como TIC's), situándose desde un paradigma pedagógico constructivista, a partir de la aplicación de la teoría de aprendizaje significativo, propuesto por Ausubel (2002), quien consideraba que el uso de organizadores previos, junto con la motivación y la repetición en diversos contextos, consolidaba más una idea en la "memoria que múltiples repeticiones de la misma idea en el mismo contexto" (p.21).

Por lo que para el diseño del AVA (Ambiente Virtual de Aprendizaje) se recrearon diversos contenidos, que incluyeron el uso de 
organizadores previos con la finalidad de acortar la distancia, entre lo que ya sabe el estudiante y lo que necesita aprender, al tiempo que se estimuló la motivación; buscando como indica Carrasco (2011): "predisponer a los alumnos a que aprendan” (p.133). Lo anterior se hizo a partir de animaciones, información en variados formatos, y la comunicación constante del profesor; a fin de que como indicaron Coll, et al. (2007), el estudiante rompiera el equilibrio cognitivo que presentaba, y realizara un contraste entre lo que ya sabe y el nuevo conocimiento.

Para la implementación de los OVAS se cumplió con conceptos de granularidad $^{1}$, y los mínimos exigidos por el MEN 2006. De ahí que por cada requisito se elaboró un objeto, el cual a partir de la construcción de un guion técnico dio cuenta de la inclusión e interacción de los contenidos, actividades, prácticas, créditos, derechos de autor, elementos teóricos y de contextualización; orientados a facilitar la navegabilidad y accesibilidad del usuario.

Una vez finalizados, se dispusieron en el AVA los OVAS junto con los demás recursos, organizados de forma hipermedia ${ }^{2}$, lo que permitió una navegación libre en la que el estudiante seleccionaba el contenido al que deseaba acceder, tal como se plasma en el mapa de navegación de todo el curso, expuesto en la figura 1.

\section{Figure 1}

Mapa de navegación del AVA

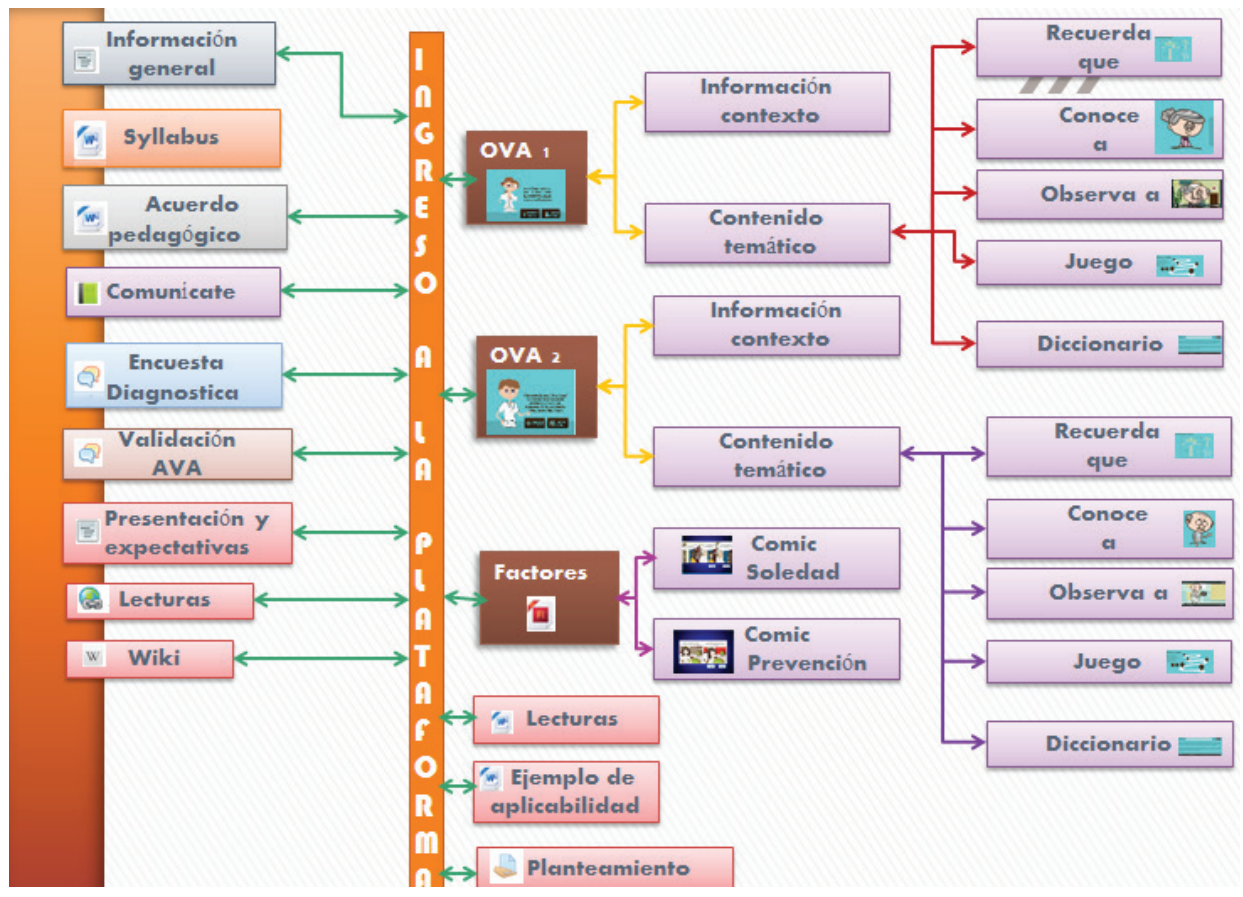

Fuente: autoras de la investigación.

1. Se asocia con el tamaño del OVA y su nivel de agregación. Entre menor sea su tamaño, es mas facil reutilizarlo.

2. De acuerdo a la clasificación de Gros (1997). 


\section{METODOLOGÍA}

La presente investigación gira en torno al proceso de atención en enfermería, específicamente a la etapa de valoración, la cual debe ser planificada, metódica, y continua; asumida con una actitud reflexiva y consiente en la que el detalle, la observación, y la inferencia son claves para lograr un cuidado de enfermería individualizado, pues no hay dos pacientes iguales en cuanto al contexto, características y condiciones propias.

Esa individualidad propia de la valoración y el acercamiento al fenómeno de aprendizaje, como consecuencia de un proceso particular de apropiación de conceptos en torno a la valoración, hacen que sea adecuado trabajar desde la investigación cualitativa. Lo anterior, porque de acuerdo a Bautista (2011) esta permite hacer un énfasis: "En la obtención de datos textuales abiertos, en las propias palabras y frases de la población local, particularmente para obtener información del contexto de conducta y de los sistemas que influyen en el comportamiento" (p. XVI).

Lo que permitiría un aumento en la comprensión del fenómeno, a partir del acercamiento a su complejidad desde distintas miradas.

Al determinar el diseño metodológico, se pudo corroborar que hay varias posturas al respecto, por lo que en estas cuestiones no se ha dicho ni se dirá la última palabra, finalmente es el sujeto investigador quien lo construye. Al respecto Sampieri lo fundamenta de la siguiente manera:

No hay dos investigaciones cualitativas iguales o equivalentes (son como hemos dicho "piezas artesanales del conocimiento" hechas a mano a la medida de las circunstancias). Puede haber estudios que compartan ciertas similitudes, pero no replicas como en la investigación cuantitativa. Recordemos que sus procedimientos no son estandarizados. Simplemente el hecho de que el investigador sea el instrumento de recolección de los datos y que el contexto o ambiente evolucione con el transcurrir del tiempo hacen a cada estudio único. (Sampieri, Fernández, \&Baptista, 2006, p.686)

De tal manera que recomienda más que centrase en el diseño garantizar la rigurosidad en el análisis de los datos, por lo que para ello en investigación se utilizó la codificación propuesta por la teoría fundamentada de Glaser (citado por Sampieri et al., 2006), quien subraya la importancia de que la misma emerja a partir de la codificación de los datos.

Vale la pena aclarar que la teoría que surge de la presente investigación es sustantiva y, por tanto su naturaleza es local, circunscrita a la población objeto de estudio y da cuenta de cómo los estudiantes relacionan su rendimiento académico con diversos factores que los profesores pueden pasar por alto.

Dada la importancia de los datos, se determinó que serían recolectados a partir de una Guía de Observación, cuyo diseño siguió los lineamientos establecidos por la técnica de Observación participante, la que según Bautista (2011), es un medio por el cual el investigador tiene una estrecha relación con el grupo a observar, lo que permite comprender o explicar mejor un fenómeno; la cual fue a su vez validada por el Doctor Burgos.

Una vez aplicada la guía durante la valoración practica en el ámbito domiciliario, los estudiantes fueron retroalimentados en torno a los resultados alcanzados y se les solicitó que a partir de un análisis introspectivo, respondieran un cuestionario autoadministrado de forma individual ${ }^{3}$, el cual estaba incluido dentro de la guía, para que a partir de preguntas abiertas explicaran cuales fueron las razones de alcanzar un determinado criterio de medición, de acuerdo a lo observado por el investigador.

Para determinar la muestra del total de la población, (constituida por un universo de 51 estudiantes matriculados en sexto semestre del

3. De acuerdo a Sampieri et al., 2006 este tipo de cuestionarios es entregado de manera individual y las respuestas las marcan los mismos participantes. 
programa de enfermería de la universidad de Cundinamarca seccional Girardot, periodo B del 2013), se aplicó un muestreo opinático ${ }^{4}$, cuya estrategia de selección fue de conveniencia, con relación a los estudiantes que tuvieran clase práctica con la profesora quien a su vez hacía las veces de investigadora.

De tal selección resultó una muestra de 26 estudiantes, los cuales fueron divididos en 4 subgrupos al azar (A, B, C, D), lo anterior por la necesidad de que durante su práctica rotaran por los servicios de medicina interna, unidad de cuidado intensivo, institucional ancianato y domicilio. Siendo este último de especial interés, ya que la investigación se focalizó en la valoración desarrollada en este ámbito.

La elección de cuales subgrupos (A, B, C, D) iban a tener acceso al aula estuvo relacionada con la demora en todos los trámites de solicitud de apoyo y el tiempo necesario para que pudieran interactuar con el aula antes del desarrollo de su práctica. Resultando así que los primeros dos (A-B) hicieron las veces de grupos control, mientras los dos restantes (C-D), fueron los grupos experimentales.

Como características generales de los estudiantes se puede mencionar que estaban entre los 18 y 25 años de edad, con un nivel académico promedio regular, residentes en zonas aledañas a Girardot como incluidos los municipios de Tocaima, Agua de Dios, Fusagasugá, Ricaurte y clasificados de acuerdo al estrato socioeconómico dentro de la clase media baja.

\section{RESULTADOS Y DISCUSIÓN}

El primer instrumento utilizado fue una evaluación diagnostica inicial ${ }^{5}$; orientada a obtener

\footnotetext{
4. "La selección de contextos y/o informantes se realiza siguiendo criterios más pragmáticos y de factibilidad, como la facilidad de contactos, la accesibilidad". (Vázquez et al., 2011, p.62)

5. La evaluación diagnostica inicial es un tipo de evaluación, cuyo objetivo es: "determinar la situación de cada estudiante antes de iniciar un determinado proceso de enseñanza /aprendizaje, para de esta manera, poder tomar conciencia de las necesidades (profesorado y alumnado) y ser capaz de adaptarse a ellas" (Jorba \& Sanmarti, 1996, p. 95). Esta evaluación favorece por tanto la autorregulación continua de los aprendizajes por parte de los estudiantes.
}

información relevante sobre actitudes, hábitos, motivaciones, habilidades tecnológicas, y grados de dominio ${ }^{6}$. Así mismo en conocimientos previos, de los requisitos universales de soledad e Interacción social y prevención de peligros para la vida, el bienestar y el funcionamiento humano, los cuales son prerrequisito para el desarrollo del núcleo temático.

A partir del análisis cualitativo de las respuestas dadas por los estudiantes, se pudo determinar que a pesar de tener un grado 2 de dominio, se encontraban altamente motivados por las expectativas de cuidado que pudieran suministra sus pacientes y que su manejo de las TIC's era el necesario para el desarrollo del curso. Posterior a ello, los grupos A-B desarrollaron su núcleo temático de forma presencial, mientras los grupos C-D además de lo anterior, tuvieron la oportunidad de acceder a un ambiente virtual. Finalmente, ambos grupos realizaron su práctica en diferentes ámbitos, siendo el domiciliario, el espacio donde fue implementada la Guía de Observación ${ }^{7}$ para cada estudiante, con el objetivo de determinar si el ambiente virtual contribuía o no en el mejoramiento de la valoración realizada por estudiantes de sexto semestre de enfermería a los dos requisitos mencionados en la pregunta problema.

Lo anterior fue observado a partir de modificaciones en la conducta y procedimiento, durante el desarrollo de la valoración práctica, lo cual fue complementado con un cuestionario autoadministrado de forma individual que permitía a los estudiantes explicar el porqué de los resultados obtenidos en dicha valoración.

El análisis de los datos recolectados, se hizo a partir de lo establecido por el diseño de la teoría fundamentada emergente descrito por de Sampieri et al. (2006), proceso que implicó

6. Según la escala de Labruffe (2008) se tienen los siguientes grados de dominio: Grado 1: dominio o práctica débil o torpeza en enumerar conocimientos o realizar prácticas. Grado 2: conocimiento dudoso o ejercicio poco hábil o ágil. Grado 3: conocimientos y prácticas adquiridos se demuestran con comodidad y fluidez. Grado 4: dominio de conocimientos y prácticas o visualización precisa. (p.43-44)

7. Guía elaborada a partir de lo descrito por Bautista (2011) para corroborar los cambios ocurridos en la práctica domiciliaria entre los 4 subgrupos A, B,C,D 
la organización y trascripción de los datos a un archivo de Excel, a partir del cual y luego de un proceso de lectura repetitiva línea a línea, permitió establecer las unidades de análisis que fueron codificadas para determinar su frecuencia. Posterior a ello, se realizó la codificación de segundo plano donde se conceptualizaron y relacionaron las categorías y subcategorías emergentes que se resumen en la figura 2:

\section{Figure 2}

Categorías y Subcategorías emergentes en la investigación

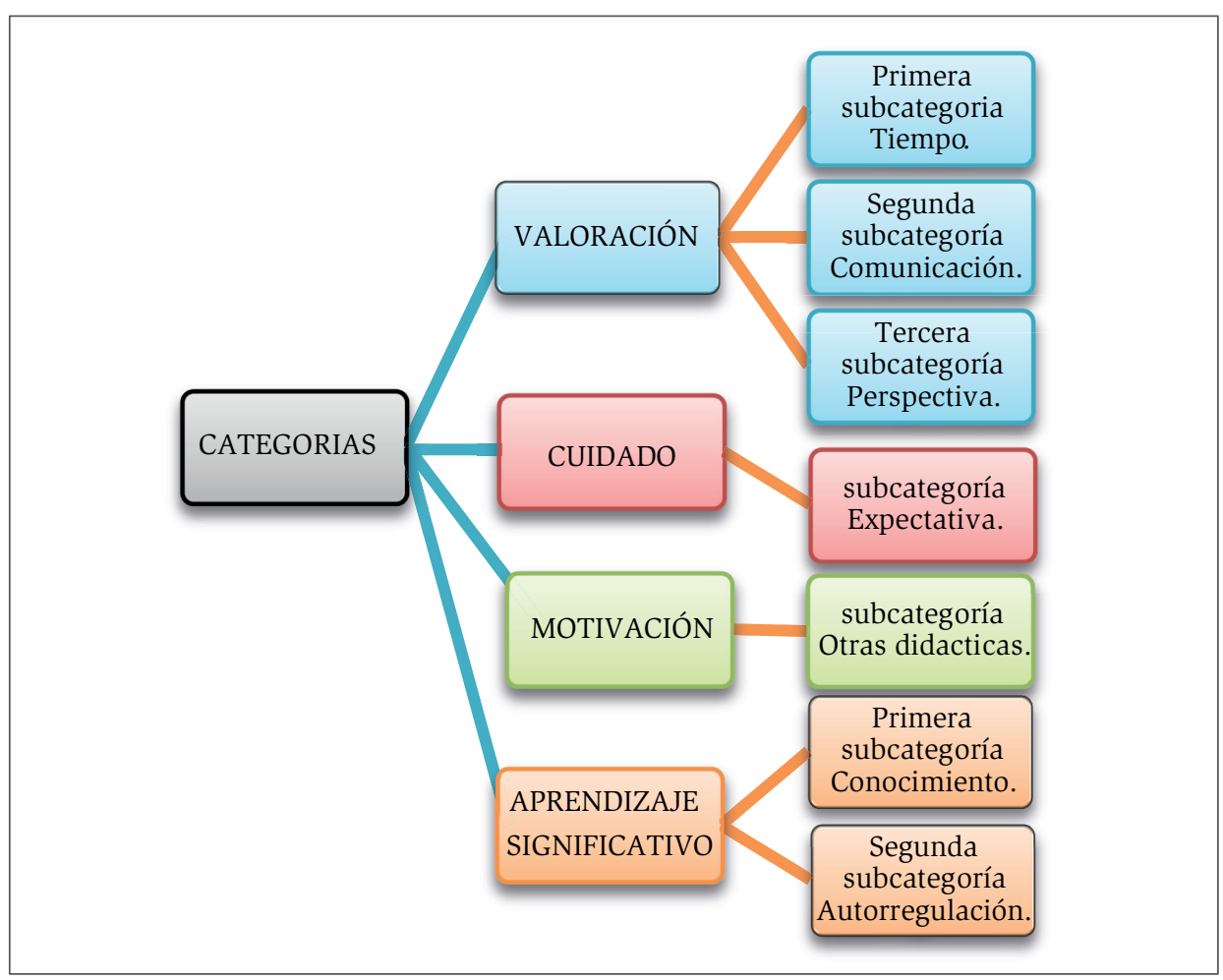

Fuente: Elaborada por las investigadoras Ruby Torres y Adriana Hernández.

En términos generales los resultados del grupo experimental C-D, muestran un grado de dominio mayor respecto de los grupos A-B, que significó una mejor valoración. ${ }^{8}$ Ello debido a que de acuerdo con lo mencionado por los estudiantes, el uso de las TIC's los motivó y promovió el uso flexible del tiempo, que fue percibido por los estudiantes del grupo C-D desde un punto de vista kairotico $^{9}$, impulsándolos a invertir más horas de trabajo independiente, que como consecuencia implico una mayor profundización en los conocimientos y permitió que sus valoraciones tuvieran una perspectiva más profunda en el requisito de Soledad e Interacción social al no conformarse con las preguntas que estaban indicadas en el formato. Por otro

8. Etapa inicial del proceso de atención de enfermería, donde el enfermero(a), mantiene una relación efectiva con el paciente, familia y otros para recolectar datos relacionados con la salud y colaborar continuamente, en la identificación en conjunto de la influencia biológica, social, psicológica y cultural en la capacidad de autocuidado del paciente.

9. Kairos es la medida cualitativa del tiempo, donde se cuentan son las experiencias. 
lado, los estudiantes de los grupos A-B que solo realizaron la valoración desde una perspectiva superficial, expresaron no tener los suficientes conocimientos, ni el tiempo necesario, lo que se pone en evidencia con las siguientes expresiones: Muy corto el tiempo para profundizar en la valoración y el cuidado"10, "El trabajo independiente para profundizar en este requisito fue muy corto en tiempo"11 "Fue la primera vez que puse en práctica los conocimientos adquiridos para poder valorar este requisito"12, "Fue muy corto el tiempo para continuar valorando y planteando más cuidados"13. Lo que muestra que para el estudiante es difícil "Aplicar aquello que no conoce mientras intenta conocerlo, pudiendo obtener un aprendizaje parcial y no global como seria lo deseado desde el punto de vista docente" (Falco, 2009, p.71).

Con relación a la comunicación, y su incidencia en la valoración, se pudo establecer que si bien los estudiantes en su totalidad tienen claridad sobre su relevancia en la valoración, algunos no lograron hacer un despliegue de manera específica en el requisito de prevención, por circunstancias variadas, que iban desde la inseguridad al estar acompañados por el profesor, hasta el considerar que con solo la observación de algunos factores se podría igualar la recolección de datos que se obtienen durante la comunicación y la valoración física. Lo anterior se expone en los siguientes comentarios: "brindar buena empatía y comunicación con el adulto mayor y familia como estrategia para la valoración"14, "La usuaria era corta de palabras y decía poco por eso solo respaldó su valoración del requisito en lo observado en alguno de estos factores humanos"15. Lo cual permite inferir, que al ser la comunicación un proceso multidimensional muy complejo tal como lo mencionan (Cibanal, Arce, \& Carballal, 2010). Los estudiantes pueden no tener en cuenta todos los aspectos que involucra y por tanto desarrollarla de forma parcial.

10. Guía Orientadora, estudiante 3 del grupo A.

11. Guía Orientadora, estudiante 2 del grupo A.

12. Guía Orientadora, estudiante 2 del grupo B.

13. Guía Orientadora, estudiante 3 del grupo B.

14. Guía Orientadora, estudiante 1 del grupo A

15. Guía Orientadora, estudiante 1 del grupo C.
Respecto a la segunda categoría cuidado ${ }^{16}$,el éxito del mismo, además de estar directamente relacionado con la valoración realizada, fue determinado por las diversas expectativas que tuvieron los estudiantes; siendo prioridad para los grupos A-B, el mejoramiento de la calidad de vida a través de cuidados enfocados a la necesidad del paciente de manera individualizada. Mientras que los grupos C-D, orientaron su actuar en diversos focos, tales como la educación, la escucha activa de acuerdo a las necesidades del paciente, buscando el enlentecimiento de la enfermedad; a través del autocuidado desembocando en mejores planes de cuidado.

Como relación a la motivación, mientras para los grupos A-B fue poco estimulada y como sugerencia recomendaron utilizar alguna didáctica innovadora; fue transformada para los grupos C-D con la inclusión de las TIC's, pues les permitió profundizar en el conocimiento y tener en cuenta factores que de otro modo no hubieran contemplado dentro de dichos requisitos. Lo cual queda en evidencia en los siguientes comentarios: "lo dispuesto en la plataforma me ayudo para realizar una buena valoración"17, "el ambiente virtual nos da una visualización de aspectos que antes no se creian tan relevantes como para ser interferencia en este requisito." 18 , "Fue con ayuda del ambiente virtual, allí encontré actividades muy didácticas donde se facilitaba el aprendizaje"19. Al respecto Zambrano (2012) considera que: "Las TIC'S, son herramientas que permiten reforzar la motivación del estudiante en los métodos utilizados para lograr aprender" (p.40).

Finalmente y como cuarta categoría emerge el aprendizaje significativo, el cual según los resultados obtenidos por los estudiantes de los grupos experimentales $\mathrm{C}$-D, se deduce que fue alcanzado ya que los estudiantes relacionaron la nueva información del núcleo temático, con

16. Entendido como una interacción recíproca, producto de la valoración y expresión de la práctica entre el estudiante de enfermería y el adulto mayor en el contexto domiciliario, a través de actividades dirigidas a apoyar, acompañar en el fomento del autocuidado y el mantenimiento de su salud, que en fin último se convierte en la expectativa con la que se brinda esta atención. (Duque, 1999).

17. Guía Orientadora, estudiante 5 del grupo D.

18. Guía Orientadora, estudiante 4 del grupo C.

19. Guía Orientadora, estudiante 7 del grupo C. 
los cambios normales del envejecimiento y la profundización en la valoración de los requisitos, junto a los conocimientos previos relacionados con estos temas almacenados en su estructura cognitiva, lo que es consecuencia de una actitud favorable y del apoyo de un ambiente virtual como complemento no solo de un proceso de aprendizaje, sino de autorregulación ${ }^{20}$. Hechos que promovieron el aprendizaje significativo en este grupo de estudiantes, debido a que como lo sostuvo Ausubel (2002) la estructura cognitiva se hizo clara, estable y se organizó adecuadamente, permitiendo que aparecieran significados precisos e inequívocos, en la valoración de los dos requisitos tendientes a retenerse y estar disponibles cuando el estudiante requiera transferirlos a distintos contextos. Al respecto enunciaron: "El desarrollo del PAE $E^{21}$ fue dado por el soporte teórico ofrecido por el aula y demás documentos"22, "Siento que en este momento se me facilita mucho más la planeación"23, "los cuidados de enfermería se le brindan al adulto mayor y a la familia con mayor seguridad y apropiación teórica" 24, "el ambiente virtual nos brindó ejemplos en cuanto a casos clínicos y los cuidados que se deben brindar" ${ }^{25}$, "Tuve en cuenta todos los criterios para las diferentes actividades que deben realizarse, llevando asi a que la usuaria tenga una recuperación" 26 , en la autorregulación el estudiante expresa "considero que valore gran parte de lo que tenía propuesto"27

Si bien es necesario desarrollar procesos de autorregulación más estructurados en los estudiantes, la ausencia de la evaluación que se tenía en el AVA, junto a las otras categorías y subcategorías pudo incidir en que los estudiantes del grupo A-B, estuvieran limitados a la realización de las actividades solicitadas por el docente. En

20. Woolfolk (2010) indica que mediante la conjunción, de habilidades de aprendizaje académico y las de autocontrol, el aprendizaje debe ser más sencillo para los estudiantes, al incluir el uso de diferentes estrategias académicas que les permitan obtener mejores resultados; siempre y cuando hagan uso del conocimiento de sí mismos, de las tareas y estrategias de aprendizaje, que los motiven para aprender.

21. PAE: Plan de atención de enfermería

22. Guía Orientadora, estudiante 2 del grupo C.

23. Guía Orientadora, estudiante 4 del grupo D.

24. Guía Orientadora, estudiante 7 del grupo C.

25.Guía Orientadora, estudiante 7 del grupo C.

26. Guía Orientadora, estudiante 3 del grupo D.

27. Guía Orientadora, estudiante 1 del grupo C. particular se identifica, qué frente a los resultados, los estudiantes se muestran conformes y en algunos casos orgullosos de los mismos, lo que permite señalar una indiferencia total ante la importancia de adquirir mayores niveles de dominio de conocimiento durante su proceso de aprendizaje, ya que lo que les interesa es pasar. Tal hecho finalmente tiene un efecto significativamente negativo sobre el aprendizaje del estudiante, al demostrar que el estudiante no está desarrollando la capacidad para autodirigir sus esfuerzos hacia el logro de las metas de aprendizaje.

\section{CONCLUSIONES}

El diseño e implementación de un ambiente b-learning en el núcleo temático cuidado de enfermería en la adultez y senectud, que incluyo procesos de interacción tales como animaciones, lecturas, juegos y otras actividades al interior de los dos OVAS, junto con un caricaturas y otros recursos contenidos en el AVA, aportó significativamente en el aprendizaje de la valoración de los requisitos universales de autocuidado de soledad e interacción social y prevención de peligros para la vida, el bienestar y el funcionamiento humano. Lo anterior fue evidenciado en el desempeño y expuesto en las voces de los mismos estudiantes de sexto semestre, además que les permitió fortalecer la comunicación directa en la relación de cuidado con el paciente y en la trasferencia de sus aprendizajes al contexto de la valoración y el cuidado domiciliario al adulto mayor. Lo anterior dio cuenta del primer objetivo específico en el que se propuso: Diseñar un ambiente B-learning con 2 OVAS, utilizando como estrategia didáctica el uso de animaciones y organizadores previos. Respecto al segundo objetivo específico, en el que se propuso: Interpretar posibles asociaciones entre las categorías establecidas a partir de la triangulación se concluyó que:

Con relación a las expectativas iniciales de los estudiantes, orientadas al cuidado del adulto mayor, a partir de mejorar su calidad de vida y sus procesos de rehabilitación, se debe indicar que solo fueron alcanzadas por aquellos estudiantes que lograron desarrollar una comunicación eficaz y participativa, en medio del respeto y el saber 
escuchar al otro, basados en la premisa: "quien escucha es un lector de indicios” (Vásquez, 2009, p.114). Además porque con el solo escuchar al paciente, se pueden generar cambios positivos en el estado de ánimo y la actitud hacia las condiciones de salud, convirtiéndose en un cuidado mucho más efectivo que el apoyo tangible. Al respecto Echeverría (2003) indica que las conversaciones son un instrumento con el que se pueden modificar los estados de ánimos de las personas. Permite utilizar el leguaje como un dominio de acción sobre un estado emocional y a la inversa.

De acuerdo con lo expresado, por los estudiantes del grupo experimental, el ambiente virtual fue una herramienta útil para la ampliación del conocimiento, que favoreció habilidades de autorregulación y pensamiento crítico, para apoyar la perspectiva de profundidad e integralidad en la valoración. Resultados similares a los encontrados por Consuegra \& Herrero (2012), quienes en su investigación de tesis encontraron, que la actividad independiente mediada por las TIC's, se volvió más didáctica, amena y permitió al estudiante hacerse responsable sobre el uso del espacio y el tiempo para su aprendizaje.

No obstante lo anterior, se evidenció en los estudiantes de enfermería una resistencia a la interacción virtual, restringiéndose únicamente a la entrega de tareas, tal vez porque consideraron mucho más productivo en su proceso de aprendizaje, la comunicación y retroalimentación diaria que podían tener con el profesor, y sus compañeros de grupo. Este aspecto pedagógico debe ser abordado desde el mismo currículo en enfermería, al implementar estrategias relacionadas con la virtualidad. Resultado que los ubica de acuerdo con Sigales (2004), en un uso de las TIC's con preferencia hacia el grado presencial, por lo menos en cuanto a lo relacionado con la comunicación, por ser jóvenes que se inician en sus estudios de grado, con mayor disponibilidad de tiempo y residentes cercanos al campus universitario, que al tener contacto a diario con la docente prefieren interactuar con ella presencialmente.

Con relación al profesor, para los estudiantes de ambos grupos, sigue siendo el mediador principal del conocimiento, situándolo incluso por encima del Internet, y de los libros. Concepción que solo se modificó un poco en los grupos C-D, durante el desarrollo del núcleo, por lo que sería aconsejable, incluir modelos de aprendizaje autorregulado, para que tal como señaló Woolfolk (2010), sean conscientes de sus capacidades y limitaciones a fin de vigilar de forma autónoma su proceso de aprendizaje a lo largo de la vida. En tal caso el rol del profesor debe estar orientado a ser un apoyo del estudiante, en cuanto a la búsqueda de estrategias que le sirvan como soporte, para su proceso de autorregulación, a la vez que lo motiva y guía en el desarrollo de su fuerza de voluntad.

Finalmente y para dar respuesta al tercer objetivo específico, en el que se buscó: Determinar la relación entre del uso del ambiente B-learning y las categorías de análisis que afectan el aprendizaje en los estudiantes; se concluyó que:

El ambiente b-learning, proporcionó resultados positivos en los grupos experimentales C-D en tanto el uso flexible del tiempo académico, lo convirtió en tiempo Kairotico. Tal disfrute motivo en los estudiantes un mejor aprovechamiento del tiempo de trabajo independiente, lo que se tradujo en una valoración más integral. Resulta así importante que el tiempo Kairos, sea utilizado como una estrategia pedagógica que promueva el aprendizaje significativo, pues el kairós es quien debe dar la pauta a kronos, permitiendo que el estudiante dimensione en su proceso de enseñanza, un mayor valor al instante del evento de aprendizaje de la valoración mediado por tecnologías, así como el momento único e irrepetible de la valoración y el cuidado en los dos requisitos. Al respecto afirma Hoyuelos (s.f.), que es fundamental hacer flexible la relación de Kairos y Kronos no solo en familia sino también en el aula, para lo cual resulta fundamental no programar el currículo como si los individuos aprendieran de forma estandarizada y sujeta a Kronos, lo que sin duda alguna depende de Kairos; ya que sin un tiempo de goce y de disfrute es imposible lograr un aprendizaje significativo. El ambiente B-learning, también suministró una mayor motivación, en tanto los estudiantes 
expresaron tener mejor disposición a aprender, al tiempo que indicaron que los contenidos incluidos ampliaron su visión en la valoración de los requisitos, lo que junto con el uso de la representación de diversos contextos utilizando las animación, les fue más significativo y útil a la hora de aplicarlo durante la valoración domiciliar.

Una vez resueltos los objetivos específicos propuestos al principio de la investigación, se ha dado respuesta de forma implícita al objetivo general en el que se planteó: Identificar el aporte que realiza el ambiente B- learning en los estudiantes de sexto semestre de enfermería, para la valoración de los requisitos de soledad e interacción social y prevención de peligros como parte del proceso de atención de Enfermería modelado desde la teoría de Dorotea Orem.

En tanto se comprobó que la inclusión del ambiente B-learning sí realizó un aporte positivo a los estudiantes, puesto que promovió la categoría de aprendizaje significativo a partir de la modificación de las demás categorías y subcategorías emergentes. $\mathrm{O}$ dicho en otras palabras, al sentirse motivados a partir del uso del ambiente B-learning, los estudiantes dedicaron más tiempo para ampliar sus conocimientos y por tanto sus perspectivas, lo que significó un mejor desempeño en la valoración, en tanto se sintieron más seguros al comunicarse e interactuar con el paciente y su familia; lo que les permitió tener en cuenta más elementos para realizar un plan de cuidados acertado que cumpliera con las expectativas de cuidado del adulto mayor.

Por otra parte, se evidencia que la introducción progresiva de las TIC en la disciplina de enfermería puede darse a diferentes niveles pues la revolución que han provocado en todos los ámbitos así como las expectativas de crecimiento futuro, evidencian la necesidad de formación en TIC de los futuros profesionales de la Enfermería y porque no decirlo en temas propios de la disciplina como el manejo dela teoría de Dorotea Orem, de ahí que esta investigación aporta al programa de Enfermería de la universidad y de forma más general a otros contextos dando cuentas de la necesidad de revisar varios contenidos en el currículo que pueden guiarse en el estudiante con el apoyo de las TIC.

\section{REFERENCIAS BIBLIOGRÁFICAS}

Ausubel, D.P. (2002). Adquisición y retención del conocimiento, una perspectiva cognitiva. Madrid, España: Ed. Paidós Ibérica S.A.

Bautista, N. P. (2011). Proceso de la investigación cualitativa, epistemología, metodología y aplicaciones. Bogotá, Colombia: Ed. Manual Moderno.

Carrasco, J. (2011).Enseñar hoy, didáctica básica para profesores. Madrid, España: Ed. Síntesis.

Cibanal, L., Arce, M., \&Carballal, M. (2010).Técnicas de comunicación y relación de ayuda en ciencias de la salud. Barcelona, España: Ed. Elsevier.

Coll, C. Martin, E. Mauri, T. Miras, M. Onrubia, J. Solé, I. \&Zabala, A. (2007). El constructivismo en el aula. Barcelona, España: Ed. Grao

Consuegra, M., \& Herrero, E. (2012). Propuesta metodológica para desarrollar habilidades investigativas, con apoyo de las TIC, en los enfermeros de la facultad de Lidia Doce (Tesis de Maestría).Instituto superior politécnico José Antonio Echeverría. La Habana, Cuba.

Duque, S. (1999). Dimensión del cuidado de enfermería a los colectivos: un espacio para la promoción de la salud y la producción de conocimiento. Revista investigación y educación en enfermería. 17(1). 75-85. Recuperado de http://aprendeenlinea.udea.edu.co/revistas/index.php/iee/article/ viewFile/16890/14622

Echeverría, R. (2003). Ontología del lenguaje. Recuperado de http://www.slideshare.net/ ravachol52/echeverria-rafael-ontologiadellenguaje

Falco, A. (2009) Enseñar estrategias de razonamiento y pensamiento crítico a los estudiantes de enfermería. Revista metas de enfermería, 12 (9), 68-72

Gros, B. (1997). Diseños y programas educativos. Barcelona, España: Ed. Ariel S.A.

Hoyuelos, A. (s.f.). Los tiempos de la infancia. Banco Interamericano de Desarrollo. Recuperado el 21 de mayo de 2014, de http://pub.iadb.org/uniquesigfafe58f9338cf1e1905eedf24126df11/uniquesig0/ IADBOpenPortalHomePage/

Jorba, J. San martí, N. Enseñar, aprender y evaluar: Un proceso de evaluación continua. Recuperado de http://books.google.com.co/books?id = a_rCXrBxi $\mathrm{kwC} \& \mathrm{pg}=\mathrm{PA} 95 \& \mathrm{dq}=$ evaluaci $\% \mathrm{C3} \% \mathrm{~B} 3 \mathrm{n}+$ diagno stica\&hl = es\&sa = X\&ei = I1 NmU43YDs2_sQSU1YK YAg\&ved $=0$ CDcQ6AEwAg\#v $=$ onepage $\& \mathrm{q}=$ evalu aci $\%$ C3 \% B3n \% 20diagnostica\&f $=$ false 
Labruffe, A. (2008). La gestión de competencias: planteamientos básicos, prácticas y cuadros de mando. Madrid, España: Ed.Aenor.

Sampieri, R., Fernández, C., \& Baptista, P. (2006). Metodología de la Investigación. México D.F, México: Ed. Mc Graw-Hill

Sigales, C.(2004). Formación universitaria y TIC’s: nuevos usos y nuevos roles. Revista de Universidad y Sociedad del Conocimiento. 1(1). 1-6. Recuperado de http://www.uoc.edu/rusc/dt/esp/sigales0704.pdf Scarborough general Hospital. (1984). Implementación de la teoría de déficit de autocuidado de Dorothea Orem dentro de la práctica de enfermería. Proyecto de manual para enfermeras. Preparado por: scarborough general Hospital. Departamento de Enfermería. Ontario, Canadá.. Traducido por: Cecilia Silva de Mojica RN, BNS, MEL.
Stanley, M., Blair, K., \& Gauntlett, P. (2009). Enfermería Geriátrica. MéxicoDF, México: Mc Graw Hill.

Woolfolk, A. (2010). Psicología educativa. Naucalpan de Juárez, México: Ed. Pearson.

Vásquez, F. (2009). Custodiar la Vida. Reflexiones sobre el cuidado de la cotidianidad. Bogotá, Colombia: Ed. Kimpres

Vázquez, N., Ferreira, M., Mogollón, A., Fernández, M., Delgado, M., \& Vargas,I. (2011). Introducción a las técnicas cualitativas de investigación aplicadas en Salud. Cali, Colombia: Universidad del Valle.

Zambrano, W. (2012). Modelo de aprendizaje Virtual para la educación superior. Bogotá, Colombia: Ed. ECOE 